\title{
Spontaneous intravesical knotting of urethral catheter in an infant: a rare case
}

\begin{abstract}
Urinary catheterization is common invasive procedure for several purposes on children for both diagnostic and therapeutic purposes. Though generally a safe procedure, it may cause significant morbidity if the catheter spontaneously knots inside the bladder. We report a case of spontaneous intravesical knotting of infant feeding tube in an infant which is a rare complication of urinary catheterization and treated successfully by minimal invasive technique
\end{abstract}

Keywords: urinary catheterization, catheterization complication, knotting, infant feeding tube

\author{
Volume 8 Issue 6 - 2018 \\ Venkatesh M Annigeri,' Bahubali D Gadgade, ${ }^{2}$ \\ Bhuvanesh A, ${ }^{3}$ Sameer Desai MD, ${ }^{4}$ Anil B. \\ Halgeri ${ }^{4}$ \\ 'Professor, Paediatric Surgery, SDM College of Medical Sciences \\ and Hospital, India \\ ${ }^{2}$ Associate Professor, Paediatric Surgery, SDM College of \\ Medical Sciences and Hospital, India \\ ${ }^{3}$ Professor, Anaesthesiology, SDM College of Medical Sciences \\ and Hospital, India \\ ${ }^{4}$ Urology SDM College of Medical Sciences and Hospital, India
}

Correspondence: Dr.Venkatesh MAnnigeri. Professor, and Head Dept. of Paediatric Surgery, SDM College of Medical Sciences and Hospital, Sattur, Dharwad, Karnataka, India-580009. Telephone: 91836-2477802 Fax: +91836-246165I. Email drvemkateshpgi@rediff.com

Received: November 19, 2018 | Published: December 13, 2018

\section{Introduction}

Urinary catheterization is utilized in the pediatric age group for both diagnostic and therapeutic purposes, such as urine sample collection, monitoring of fluid therapy, clean intermittent catheterization, voiding cystourethrogram. The procedure although safe, is not free of complications, it carries with it range of complications from minor like bleeding, infection to major like perforation and rarely spontaneous catheter knot. ${ }^{1}$ We report a case of intravesical spontaneous knotting of infant feeding tube in an infant, which is rare complication of urinary catheterization treated successfully by minimal invasive technique.

\section{Case history}

An eight months male infants, admitted in Pediatric department for evaluation of fever, poor feeding and lethargy of 3 days. Evaluation was performed, including complete blood cell count, blood culture, chest radiograph, and direct catheterization for urinalysis and culture using a $5 \mathrm{Fr}$ feeding tube. The nurse performing catheterization reported that she was unable to remove the catheter after insertion. Pelvis roentgenogram showing a true knot in the catheter in posterior urethra (Figure 1A). Pediatric surgeon opinion was asked, on examining along the urethra, the knotted catheter could be palpated at the perineum. Several attempts at forceful introduction of sterile saline and contrast material under fluoroscopy failed to unwind the loop. Under general anesthesia another attempt was made to untie the knot and straighten the catheter with $3 \mathrm{~F}$ ureteric catheter stylet through the catheter lumen. Failing this maneuver catheter was pushed in to bladder from posterior urethra with the help of ureteric catheter stylet (Figure 1B) Knotted feeding tube removed by minimal invasive technique from the bladder. The bladder was filled with normal saline through feeding tube, A $5 \mathrm{~mm}$ laparoscopic port was introduced in the bladder in the suprapubic region and knotted feeding tube identified. A 6-7.5 Fr ureterorenoscope was introduced through the laparoscopic port and catheter (Figure 2A and B) was removed under vision after cutting the feeding tube at penile end. After port site closed Foley catheter was inserted and was left in place for 3 days. Urinalysis showed evidence of a urinary tract infection which improved with intravenous antibiotics. Post operative course was uneventful. The child had been passing urine in good stream in follow up.
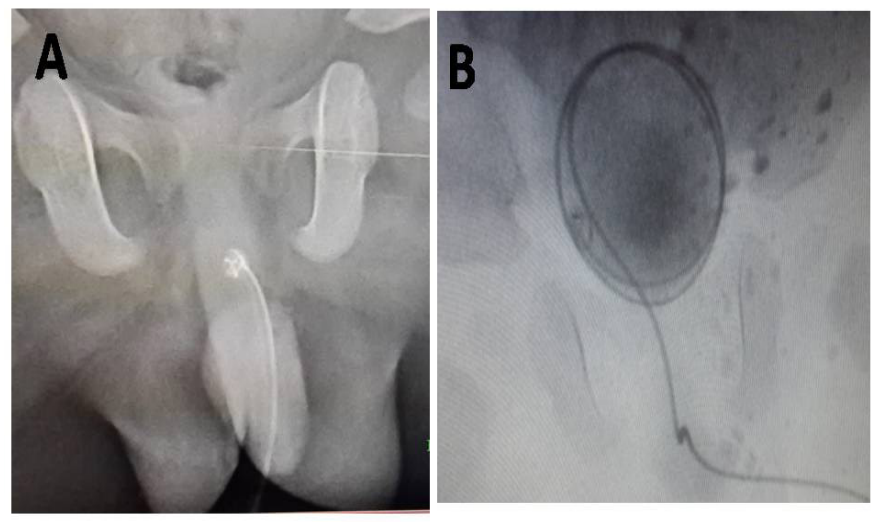

Figure IA Plain X-ray pelvis showing infant feeding knotted tube in the posterior urethra.

Figure I B Fluoroscopic view after pushing the knotted tube in to the bladder with help of with ureteric catheter stylet. 


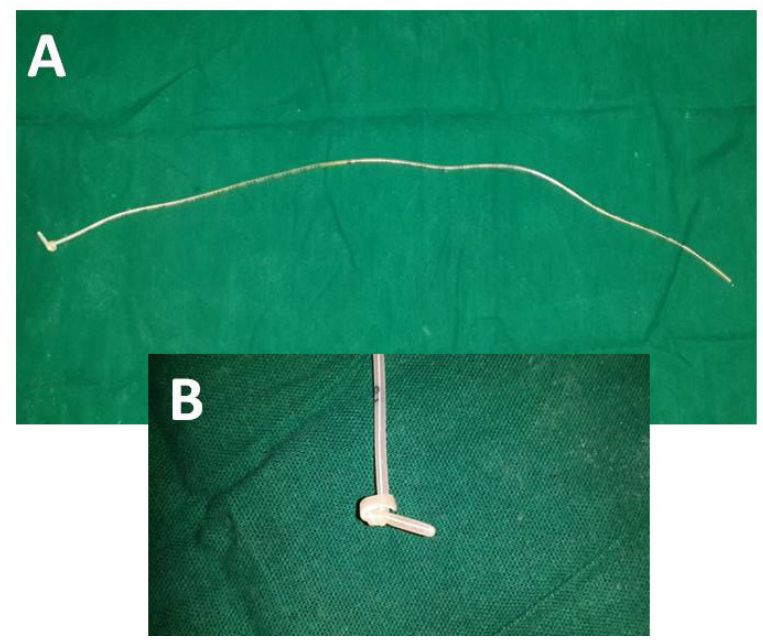

Figure 2A A Spontaneously knotted feeding tube after removal from MIS Figure 2B Close-up of the knot on the feeding tube

\section{Discussion}

Bladder catheterization is the most common day to day procedure done for monitoring of urine output, therapeutic and diagnostic purpose. Urine samples obtained by catheterization or suprapubic aspiration are the standard for diagnosis of urinary tract infection in children less than 2 years. ${ }^{2}$ Spontaneous knotting of the urinary catheter has been reported to occur in 0.2 per 100,000 catheterizations. ${ }^{3}$ Spontaneous intravesical knotting of catheters are more common in neonates and children than in adults and more commonly in males than females. ${ }^{4}$ In the present case, a complication of bladder catheterization for obtaining urine sample was encountered for short time application. Intravesical knotting has been reported catheters when left for long term bladder drainage, but also after short term such as clean intermittent catheterization, and voidingcystourethrography. ${ }^{2}$ Although knotting of urethral catheters is uncommon, pulling the catheters out may lead to severe morbidity, including complications of general anesthesia, radiation exposure, hematuria, stricture development and financial burden on the patient, in the form of investigations and additional procedures. ${ }^{3}$ A high index of suspicion is required to diagnose knotting of infant feeding tube when it cannot be withdrawn easily. This will prevent traumatic urethral injury due to forcible removal and subsequent risk of urethral stricture.

Water-current generated by the flow of urine appears to play an important role in the pathogenesis of catheter knotting. When excessive length of flexible catheter is inserted in the bladder and forms a loop; subsequently as the catheter is withdrawn a knot can form and tightens on withdrawal leading to impaction of the catheter. ${ }^{[4]}$ Risk factors identified includes catheter size less than $10 \mathrm{Fr}$, the use of feeding tube as catheter ,bladder spasm, over distended bladder and insertion of more than $10 \mathrm{~cm}$ length of catheter inside the bladder. ${ }^{4} \mathrm{In}$ the present case, risk factors includes the catheter had been inserted too far into the bladder. The catheter was $5 \mathrm{Fr}$ and feeding tube used as catheter. Recognition of the complication of catheter knotting is important. This hazard needs to be borne in mind when inserting the catheter as well as when difficulty is encountered while attempting to remove the catheter.
Prevention of spontaneous knotting of the feeding tube can be achieved by careful selection of the catheter and gaining better understanding of urethral anatomy and safe insertion lengths. The newborn male urethra measures $5 \mathrm{~cm}$, increasing to $8 \mathrm{~cm}$ by three years of age and to $17 \mathrm{~cm}$ by adulthood. The female urethra length is comparatively shorter and grows at a slower rate. It measures $2.18 \mathrm{~cm}$ at birth and increases to $2.54 \mathrm{~cm}$ by five years of age and to $3.78 \mathrm{~cm}$ in adulthood. ${ }^{5}$

Several techniques have been described to retrieve the knotted catheter. They include sustained traction under anesthesia, ${ }^{6}$ unraveling the knot using a guidewire ${ }^{7}$ endoscopic retrieval ${ }^{8}$ and suprapubic cystotomy. Sustained traction holds the risk of urethral injury; moreover, the technique is not beneficial when the knot is bulky or when two catheters knot together as in our case. Guidewire manipulation is useful only at the early stage of knot formation when the knot is not tight enough; yet, using this technique was unsuccessful in the present case. Suprapubic cystotomy has been known as a simple, cost-effective method of removal knotted bladder catheters. ${ }^{4,9,10}$ To date, this should be replaced by minimal invasive techniques such was performed in the present case.

\section{Conclusion}

Indications for urethral catheterization must be carefully considered since it is an invasive procedure. With availability of appropriate catheters and adequate knowledge and skill, this complication can be reduced to the barest minimum. If at all this clinical scenario is encountered this could be tackled by minimally invasive means.

Funding and conflicts of interest: There is no funding and conflicts of interest involved in this study.

\section{References}

1. Carlson D, Mowery BD. Standards to prevent complications of urinary catheterization in children: should and should knots. J Soc Pediatr Nurs 1997;2:37-41.

2. Kervancioglu E. A rare case presentation: intravesical catheter knotting, J Endourol Case Rep 2018; 4(1):82-83.

3. Arena B, McGillivary D, Dougherty G. Urethral catheter knotting: be aware and minimize risk. CJEM 2002;4(2):108-110.

4. Raveenthiran V. Spontaneous knotting of urinary catheters: clinical and experimental observations. Urol Int 2006;77(4):317-321.

5. Anbu AT. Urethral catheter knotting in preterm neonates. Indian Pediatr 2004;41(6):631-632.

6. Pearson-Shaver AL, Anderson MH. Urethral catheter knots. Pediatrics 1990;85(5):852-854.

7. Harris VJ, Ramilo J. Guide wire manipulation of knot in a catheter used for cystourethrography. J Urol 1976;116(4):529.

8. Dogra PN, Nabi G, Goel R. Endoscopic removal of knotted urethral catheter: a point of technique. Urol Int 2003;71(1):8-9.

9. Foster H, Ritchey M, Bloom D: Adventitious knots in urethral catheters: report of 5 cases. J Urol 1992; 148(5):1496-1498.

10. Sarin YK. Spontaneous intravesical knotting of urethral catheter. APSP J Case Rep 2011;2(23):21. 